\title{
Circulating MiR-1 Levels in Acute Myocardial Infarction and Its Significance in Differential Diagnosis and Prognosis
}

\author{
Kuzhandai Velu $\mathrm{V}^{1}$, Ramesh $\mathrm{R}^{2}$, Mohammad Hanifah ${ }^{3}$, Lenin $\mathrm{M}^{4}$
}

\begin{abstract}
Background: Acute myocardial infarction (AMI) is a condition where there is a formation of thrombus which reduces the blood flow in the coronary arteries resulting in coronary vasospasm, further leading to cardiac muscles dysfunction or even death. Quick and accurate diagnosis of AMI plays a major role in the management and prognosis of this disease. Circulating miRNA has been a promising cardiac biomarker for AMI as well as cardiovascular disease in recent years. In the circulation miR 1 overexpressed when there is any damage in the cardiac cell. A very few articles documented the use of miR-1 as a diagnostic marker in AMI. The aim of the study was to find the diagnostic and prognostic role of miR-1 in AMI.
\end{abstract}

Methodology: In this study, 62 AMI patients in the age group of 30-75 years and 30 healthy controls were included. Blood samples were collected at a different time of admission, 6 hours, 12 hours, 24 hours and 72 hours for the subject. Serum was separated immediately for the analysis of CK (NAC) and CK MB, Hs troponin T and micro-RNA-1 was quantification by RT-PCR. To study the kinetics of miR-1 repeated measure of analysis of variance (ANOVA) was analyzed. To find the diagnostic utility AUC, likelihood and odd's ration was performed.

Results: The miR-1 expression is expressed in a change in fold $27.36,57.17,43.55,32.50$ and 24.09 fold increase at the different time. The study revealed that the sensitivity $69 \%$, specificity $66 \%$, LR was 1.59 and OR 4.04 in the differential diagnosis of AMI.

Conclusion: In a total expression of miR-1 showed statistical significance in different time internal which will help us to use in the differential diagnosis of AMI.

Keywords: Acute myocardial infarction, Hs cnTn T, miR-1.

Indian Journal of Medical Biochemistry (2019): 10.5005/jp-journals-10054-0079

\section{INTRODUCTION}

A cute myocardial infarction (AMI) is most commonly encountered cause for mobility and mortality in the developing country as well as worldwide. ${ }^{1-3} \mathrm{AMI}$ is a condition where there is a formation of thrombus which reduces the blood flow in the coronary arteries resulting in coronary vasospasm, further leading to cardiac muscles dysfunction or even death. ${ }^{4,5}$ Differential diagnosis of AMI can be made as unstable angina (UA), ST-elevated myocardial infarction (STEMI) and non-ST-elevated myocardial Infarction (NSTEMI). In the past, diagnosis of AMI was based on the clinical symptoms, changes in the ECG and levels of conventional cardiac biomarker like CKMB and cardiac troponin, ${ }^{6-8}$ NSTEMI. Recently high sensitivity cardiac troponin assays were used, which eventually picks up the low concentration of cardiac troponin that helps in the early diagnostic time of AMI. Quick and accurate diagnosis of AMI plays a major role in the management and prognosis of this disease. However, there is still a clinical need for the identification of novel biomarkers with high sensitivity and specificity. ${ }^{9}$ Circulating mi RNA has been a promising cardiac biomarker for AMI as well as cardiovascular disease in recent years.

Micro-RNAs are endogenous small ( 22 nucleotides) singlestranded, non-coding RNAs that regulate gene expression at the posttranscriptional level by binding to the three prime untranslated regions (UTRs) of their target $m R N A,{ }^{10,11}$ single stranded molecules of about 22 nucleotides in length and function post-transcriptionally by partial binding (partial complementarity Though the biological functions of miRNAs are not fully understood, and it is clear that some miRs are present in tissues are cell-specific manner. A recent report shows that miRs are also present in various biological fluids including blood. The levels of specific miR are linked to the diagnosis and prognosis of the disease. Circulating miRs are more stable in blood, as well as in extreme conditions such as temperature, $\overline{1,4}$ Research Scholar and Tutor, ${ }^{2}$ Professor and Head, ${ }^{3}$ Professor

${ }^{1-4}$ Department of Biochemistry, Jawaharlal Institute of Postgraduate Medical Education and Research, Puducherry, India

Corresponding Author: Ramesh R, Professor and Head, Department of Biochemistry, Jawaharlal Institute of Postgraduate Medical Education and Research, Puducherry, India, e-mail: rameshrdr30@gmail.com

How to cite this article: Velu KV, Ramesh R, Hanifah M, Lenin M. Circulating MiR-1 Levels in Acute Myocardial Infarction and Its Significance in Differential Diagnosis and Prognosis. Indian J Med Biochem 2019;23(1):174-177.

Source of support: Nil

Conflict of interest: None

$\mathrm{pH}$ and freeze-thaw cycles ${ }^{12,13}$ The release of mi RNAs is not only a consequence of cell death or plasma membrane disruption but also an active response to ischemia. In the circulation miR-1 overexpressed when there is any damage in the cardiac cell. A very few articles documented the use of miR-1 as a diagnostic marker in AMI. In this study, we try to find the diagnostic and prognostic role of miR-1 in AMI.

\section{SUBJECTS AND METHODS}

During the annual year, 2016 (January-December) we have studied 62 AMI patients at age group 30 to 75 years and 30 healthy controls form Mahatma Gandhi Medical College and Research Institute a tertiary healthcare center, Puducherry in collaboration with General Medicine Unit. The final diagnosis of AMI was based on the European Society of Cardiology/American College of Cardiology guidelines. All the study subjects were included after obtaining Institutional Human Ethics Committee approval and informed consent from the subjects.

(O) The Author(s). 2019 Open Access This article is distributed under the terms of the Creative Commons Attribution 4.0 International License (https://creativecommons. org/licenses/by-nc/4.0/), which permits unrestricted use, distribution, and non-commercial reproduction in any medium, provided you give appropriate credit to the original author(s) and the source, provide a link to the Creative Commons license, and indicate if changes were made. The Creative Commons Public Domain Dedication waiver (http://creativecommons.org/publicdomain/zero/1.0/) applies to the data made available in this article, unless otherwise stated. 


\section{Laboratory Data}

Venous blood samples were collected in clot activator vacutainers at the time of admission, 6 hours, 12 hours, 24 hours and 72 hours for the subject. Serum was separated immediately for the analysis of CK (NAC), and CK MB was done by autoanalyzer and Hs troponin $\mathrm{T}$ (Hs-cnTn T) was estimated using fully automated chemiluminescence analyzer (Roche Diagnostics-COBAS e411) and the same day. The leftover serum sample was aliquoted in $1.8 \mathrm{~mL}$ cyro tube and stored in $-20^{\circ} \mathrm{C}$ until the time of micro-RNA analysis. All the estimation was subjected to stringent quality control.

\section{MicroRNA Analysis}

Total RNA was isolated from serum using a mini spin column kit (Helini biomolecules, India) according to the manufacturer's instruction. From that cDNA was synthesized using cDNA synthesis kit (Helini biomolecules, India), as per documented specification. Quantification by RT-PCR was carried out in $25 \mu \mathrm{L}$ using Helini Pin the Tail Probe System method, according to the protocol of the manufacturer (Helini, Biomolecules P. Ltd, India).

\section{Statistical Analysis}

All the data were entered transcript to the excel sheet. Shapiro Wilk test was performed to find whether the data followed a normal distribution or not. Data were expressed in mean and standard deviation. Student ' $t$ ' test was performed to find the significant difference between the groups. Kinetic studies of miR and cardiac biomarker were analyzed by repeated measures of ANOVA. For evaluating the diagnostic accuracy ROC, LR, PPV, and NPV were calculated. All the statistical analysis was done using Epi Info version 4.0.2.

\section{Results}

\section{Baseline Character of AMI Patients}

Among 62 patients, 49 were males and 13 were females and their age group was between 30 to 70 years. Admission sample of AMI patients, serum cardiac biomarker (CK-MB,hsCnTn T) which showed a significant difference between and among the group as depicted in Table 1.

\section{Kinetics of Circulating MiR-1 Levels in AMI}

MiR-1 expression in the serum was analyzed by using RT-PCR, and the result was summarized in Table 2 and Graph 1. The miR-1 expression is expressed in a change in fold 27.36, 57.17, 43.55, 32.50 and 24.09 fold increase at different time intervals 0 hour, 6 hours, 12 hours, 48 hours, and 72 hours, respectively. Repeated measures of ANOVA analysis was done to study the kinetics of miR-1 in the different time interval. MiR-1 was up-regulated at 4 hours when compared with another time interval. Simultaneously, we have estimated the levels of a cardiac biomarker such as CK-MB and hscnTn T. Interestingly, both the cardiac biomarker peaks at 6 hours but miR-1 peaks at 4 hours which is an early phase of AMI, could help us in the early diagnosis.

\section{Diagnostic Utility of miR-1 in AMI}

ROC curve was constructed for miR-1 between AMI patients and healthy volunteers for the evaluation of diagnostic accuracy. The ACU for miR-1 at different time interval were as follows: $0.954,0.872$, $0.877,0.841$ and 0.882 , respectively (Table 2 and Graph.1). In addition to that, we have subgroup AMI into STEMI and NSTEMI and plotted ROC curve for miR-1 to find out the role in the differential diagnosis results are depicted in Table 2 and Graph 2. The study revealed that the sensitivity was 69 , specificity was 66 , the positive predictive ratio was 76.36 , Negative likelihood ratio was 44.44 , likelihood ratio was 1.59 and diagnostic odd ratio 4.04 (95\% Cl: 1.72-9.51) in Table 3.

\section{Discussion}

MiRNAs are a group of non-coding RNAs that regulates normal physiological gene expression, and it gets affected and released into the circulation during the pathophysiological condition of various diseases. ${ }^{10}$ Studies documented miR response to cardiovascular diseases. There is quite a few number of miRs are a profile in AMI patients, one among those which has frequently studied was miR-1. miR-1 involved in the cardiogenesis and controls proliferation and differentiation for normal cardiac chamber development. It was upregulated when there is cardiac cell injury or necrosis. ${ }^{14}$ Since the biochemical basis of the release of miR-1 into the circulation from the heart after AMI still was not clearly defined, so we aimed to study the diagnostic and prognostic role in AMI. The result shows

Table1: Basic clinical characteristics of controls and AMI patients

\begin{tabular}{llll}
\hline & & \multicolumn{1}{c}{ AMI $(\mathrm{n}=62)$} \\
\cline { 2 - 4 } Characteristics & Control $(\mathrm{n}=30)$ & $56.13 \pm 14.86$ & NSTEMI $(\mathrm{n}=23)$ \\
\hline Age & $53.24 \pm 11.56$ & $32(82)$ & $18(76)$ \\
Male, $\mathrm{n}(\%)$ & $15(50)$ & & $6(26)$ \\
History & & $13(33)$ & $9(39)$ \\
Smoking, $\mathrm{n}(\%)$ & $7(23)$ & $13(34)$ & $6(26)$ \\
Diabetes, $\mathrm{n}(\%)$ & $4(13)$ & $18(45)$ & $50.87 \pm 38.73$ \\
Hypertension, $\mathrm{n}(\%)$ & $11(36)$ & & $214.78 \pm 167.66$ \\
Serum markers & & $83.44 \pm 53.73$ & \\
CK MB (u/L) & $28.34 \pm 17.85$ & $404.06 \pm 518.33$ & \\
Hs CnTn T (ng/L) & $47.19+25.59$ & & \\
\hline
\end{tabular}

Table 2: AUC of miR-1 in acute myocardial infarction at different time interval

\begin{tabular}{llllll}
\hline & Admission & 6 hours & 12 hours & 48 hours & 72 hours \\
\hline AMI vs Control & 0.954 & 0.999 & 0.988 & 0.901 & 0.862 \\
STEMI vs NSTEMI & 0.679 & 0.614 & 0.636 & 0.643 & 0.676 \\
\hline
\end{tabular}




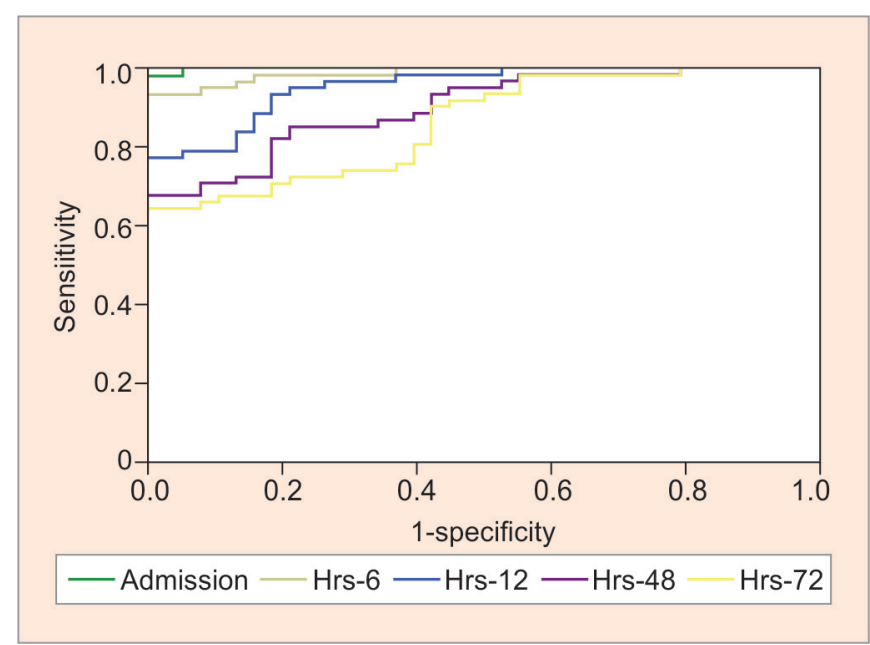

Graph 1: AUC of miR-1 between AMI vs control at different time interval

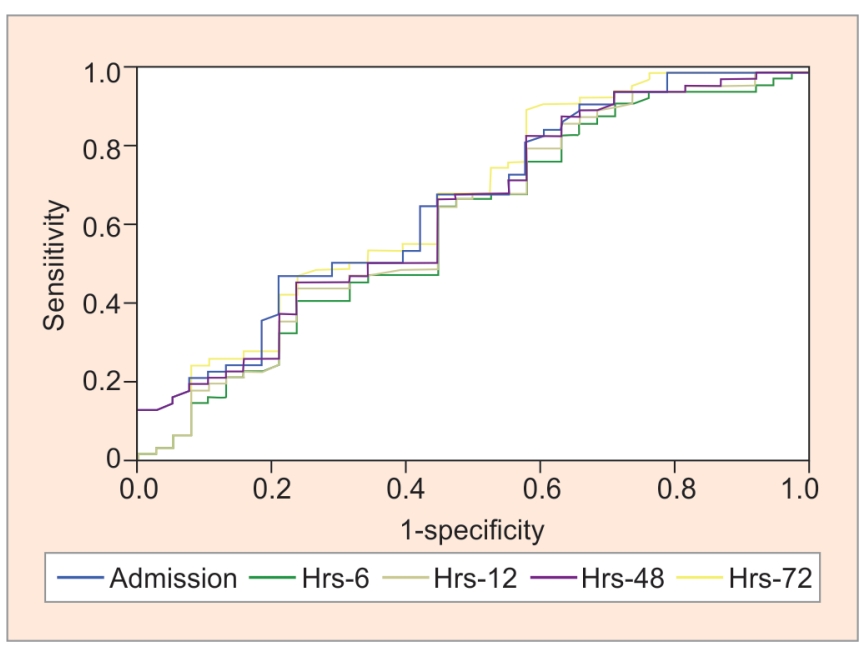

Graph 2: AUC of miR-1 between STEMI vs NSTEMI at different time interval

Table 3: Hn CnT and miR-1 and their differential diagnostic valite of myocardial infarction (STEMI and NSTMEI)

\begin{tabular}{lll}
\hline Characteristic & $H n C n T$ & miR-1 \\
\hline Sensitivity & 67 & 69 \\
Specificity & 64 & 66 \\
Positive predictive value & 73.58 & 76.36 \\
Negative predictive value & 51.06 & 44.44 \\
Likelihood ratio & 1.71 & 1.59 \\
Odd's ratio & 2.91 & 4.04 \\
& $(95 \% \mathrm{Cl}: 1.26-6.71)$ & $(95 \% \mathrm{Cl}: 1.72-9.51)$ \\
\hline
\end{tabular}

that the serum concentration of miR-1 (which is specified in the heart) increased in AMI. Circulating miR-1 was hardly detected in the healthy individual. A rise in miR-1 was evident at the early stage of AMI at the time of admission and reached a peak within 6 hours. ${ }^{15}$ In addition, miR-1 gets into the peak before other two biomarkers hscnTn T and CK-MB. D' Alessandra documented increased levels of miR-1, six hours after coronary artery occlusion in mice and also few other studies recorded significantly higher levels. ${ }^{16}$

Increased miR-1 showed a positive correlation with CK-MB by Chengal et.at. Similiarly, Ganugnwn et al. in his study showed a good correlation of miR-1 with classical biomarkers of myocardial infarction. In that same study they have reported, high sensitivity and specificity for miR-1 at different time interval 4 hours, 8 hours, 12 hours, 24 hours and 72 hours in AMI patient when compared with controls. miR-1 was associated with prolonged QRS interval. ${ }^{17}$ Our study also recorded a similar type of result when expression of miR-1 compared with novel markers of AMI.

Even now researches are in search of a suggorate biomarker for acute myocardial infarction. Suggorate markers are defined as a substance with high sensitivity and specificity and need to help in early detection of diseases or clinical condition. Certainly, miR-1 have high sensitivity and specificity which make it a novel marker but not as a suggorate maker because currently, we are using qRT-PCR for estimating which increase the turnaround time.

\section{CONCLUSION}

To conclude, there was a significant difference in the expression of circulating miR-1 in a time-dependent manner in AMI patients. With this, we can put forth that circulating miR-1 likely to be used, as a novel biomarker for differential diagnosis of acute myocardial infarction.

\section{References}

1. Ferreira GMT de M, Correia LC, Reis $\mathrm{H}$, et al. Maior letalidade e morbidade por infarto agudo do miocárdio em hospital público, em Feira de Santana-Bahia. Arq Bras Cardiol 2009 Aug;93(2): 97-104.

2. Roger VL. Epidemiology of Myocardial Infarction. Med Clin North Am. 2007 Jul;91(4):537-552.

3. Carvalho G, Rassi S. The Prognostic Value of CK-MB in Acute Myocardial Infarction in Developing Countries: A Descriptive Study. Angiol Open Access [Internet]. 2016 [cited 2017 Nov 3];4(3). Available from: http:// www.esciencecentral.org/journals/the-prognostic-value-of-ckmb-inacute-myocardial-infarction-in-developingcountries-a-descriptivestudy-2329-9495-1000183.php?aid=79371

4. Slavich M, Patel RS. Coronary artery spasm: Current knowledge and residual uncertainties. Int J Cardiol Heart Vasc 2016 Jan 23;10: 47-53.

5. Chandrasekaran B, Kurbaan AS. Myocardial infarction with angiographically normal coronary arteries. J R Soc Med 2002 Aug;95(8):398-400.

6. Kumar A, Cannon CP. Acute Coronary Syndromes: Diagnosis and Management, Part I. Mayo Clin Proc 2009 Oct;84(10):917-938.

7. Al-Hadi HA, Fox KA. Cardiac Markers in the Early Diagnosis and Management of Patients with Acute Coronary Syndrome. Sultan Qaboos Univ Med J 2009 Dec;9(3):231-246.

8. Daubert MA, Jeremias A. The utility of troponin measurement to detect myocardial infarction: review of the current findings. Vasc Heal Risk Manag 2010;6:691-699.

9. Cheng Y, Tan N, Yang J, et al. A translational study of circulating cellfree microRNA-1 in acute myocardial infarction. Clin Sci Lond Engl 1979. 2010 Apr 20;119(2):87-95.

10. Felekkis K, Touvana E, Stefanou C, et al. microRNAs: a newly described class of encoded molecules that play a role in health and disease. Hippokratia 2010;14(4):236-240. 
11. Cannell IG, Kong YW, Bushell M. How do microRNAs regulate gene expression? Biochem Soc Trans. 2008 Dec;36(Pt 6):1224-1231.

12. Glinge $C$, Clauss $S$, Boddum K, et al. Stability of Circulating BloodBased MicroRNAs - Pre-Analytic Methodological Considerations. PLoS One [Internet]. 2017 Feb 2;12(2). Available from: https://www. ncbi.nlm.nih.gov/pmc/articles/PMC5289450/

13. Viereck J, Thum T. Circulating Noncoding RNAs as Biomarkers of Cardiovascular Disease and Injury. Circ Res. 2017 Jan 20;120(2):381-399.

14. Ai J, Zhang R, Li Y, Pu J, Lu Y, Jiao J, et al. Circulating micro RNA-1 as a potential novel biomarker for acute myocardial infarction. Biochem Biophys Res Commun. 2010 Jan 1;391(1):73-77.
15. Chen $X, B a Y, M a L$, et al. Characterization of microRNAs in serum: a novel class of biomarkers for diagnosis of cancer and other diseases. Cell Res. 2008 Oct;18(10):997-1006.

16. D'Alessandra Y, Devanna P, Limana F, et al. Circulating microRNAs are new and sensitive biomarkers of myocardial infarction. Eur Heart J. 2010 Nov;31(22):2765-2773.

17. Xinran XuFD. The Role of miR-1 in the Heart:From CardiacMorphogenesis to Physiological Function. Hum Genet Embryol [Internet]. 2014 [cited 2017 Nov 29];04(01). Available from: https://www.omicsonline.org/openaccess/the-role-of-mir-in-the-heart-from-cardiac-morphogenesis-tophysiological-function-2161-0436.1000119.php?aid=26295 